\title{
T cell subsets and disease progression after total lymphoid irradiation in chronic progressive multiple sclerosis
}

\author{
R TROIANO, ${ }^{*}$ C DEVEREUX, $\ddagger$ J OLESKE, $\dagger$ T DENNY, $\dagger$ M HAFSTEIN,* \\ G ZITO, ${ }_{\S}$ C ROHOWSKI-KOCHAN,* P C DOWLING, $\S$ S DCOOK*§
}

From the Departments of Neurosciences* and Pediatrics, $\dagger$ UMDNJ-New Jersey Medical School, Newark, N.J., Clara Maass Medical Center, Belleville, N.J., $\ddagger$ and Neurology Service, VA Medical Center, East Orange, N.J.,§ USA

SUMMARY T lymphocyte subset percentages were determined in 16 total lymphoid irradiation (TLI) treated and 18 sham treated control patients with chronic progressive multiple sclerosis. During the first year after treatment, the ratio of $\mathrm{T}$ helper/inducer to $\mathrm{T}$ suppressor/cytotoxic cells (Th/Ts ratio) was significantly higher in sham treated multiple sclerosis patients who worsened clinically compared with TLI treated and sham treated multiple sclerosis patients who remained clinically stable. TLI caused a fall in the percentage of T helper cells in treated patients, while the percentage of $T$ suppressor cells remained stable during the first year after treatment. In contrast, the percentage of $\mathrm{T}$ suppressor cells fell in sham treated multiple sclerosis patients who worsened clinically.

Total lymphoid irradiation (TLI) has proven to be an effective and relatively safe method of inducing sustained immunosuppression in patients with inflammatory diseases of putative autoimmune aetiology. ${ }^{1-7}$ In these patients, TLI has caused a significant decrease in the absolute lymphocyte count, and the percentage of helper/inducer $T$ cells, with a decreased ratio of helper to suppressor $T$ lymphocytes (Th/Ts ratio) which has persisted for up to 4 years following radiotherapy. ${ }^{7}$

In this report, we present the results of determinations of T lymphocyte subsets in TLI treated chronic progressive multiple sclerosis patients and their sham treated controls.

\footnotetext{
Address for reprint requests: Raymond Troiano, MD, Chief, Division of Neurology, UMD-University Hospital, 150 Bergen Street, Newark, NJ 07103, USA.
}

Received 8 December 1987 and in revised form 29 February 1988. Accepted 7 March 1988

\section{Patients and methods}

The design of the multiple sclerosis TLI study has been described in detail elsewhere. ${ }^{8}$ In a double blind study, 40 patients with chronic progressive multiple sclerosis were randomised into either a TLI treatment group or a control group treated with a sham TLI protocol. Patients were well matched for sex, age and duration of disease.

Immediately before treatment the patients were scored clinically on a practical functional scale. Following treatment the patients were evaluated and scored at monthly intervals for 6 months and then every 3 months. Immediately before treatment and periodically after treatment, blood was obtained to measure $T$ lymphocyte helper and suppressor subsets. Samples were missed in patients who entered the study very early before lymphocyte testing was begun and in patients who missed appointments or were dropped out of the study because of severe progression or other reasons which are discussed in a previous detailed report. ${ }^{8}$ Informed consent was obtained from each subject after the nature of the study was fully explained.

Patients in each treatment group were further subdivided according to their clinical course during the first year after treatment. Patients who were clinically unchanged or who 
did not worsen severely enough to change at least one point on the functional scale were classified as stable. Patients were classified as clinically worse if they progressed severely enough to change one or more points on the functional scale. All statistical correlations and probability estimates are derived from $t$ tests of the means, two tailed.

Phenotypic determination of mononuclear cell populations were determined by reacting monoclonal antibodies (Becton-Dickinson Immunocytometry Systems, Mountainview, CA) to CD3 (LEU-4), CD4 (LEU-3), and CD8 (LEU2) receptors.

Initial analysis of patient samples for immunofluorescence were determined by fluorescent microscopy using an Olympus microscope and counting 200 cells to determine the percent positive cells under $40 \mathrm{X}$ objective. Towards the end of the study a FACS $\mathbf{4 4 0}$ cell sorter was installed and flow cytometric analysis was employed for the remaining studies. The transition to flow cytometric analysis was distributed equally across the clinical subgroups.

\section{Results}

By the end of the first year, two of the TLI patients and 11 of the control patients in the $T$ cell analysis subgroups had worsened severely enough to decline one or more points on the functional scale. The two TLI patients who worsened did not have a sufficient number of $\mathrm{T}$ cell determinations to justify inclusion as a subgroup in the statistical analysis. Therefore, only one TLI subgroup consisting of 14 TLI treated patients who remained clinically stable during the first year post treatment and two sham subgroups (stable and worse), are included in the statistics.

During the first year after treatment $\mathrm{Th} / \mathrm{Ts}$ ratios were signficantly higher in the group of sham control patients who worsened clinically compared with either the group of TLI treated patients or sham controls

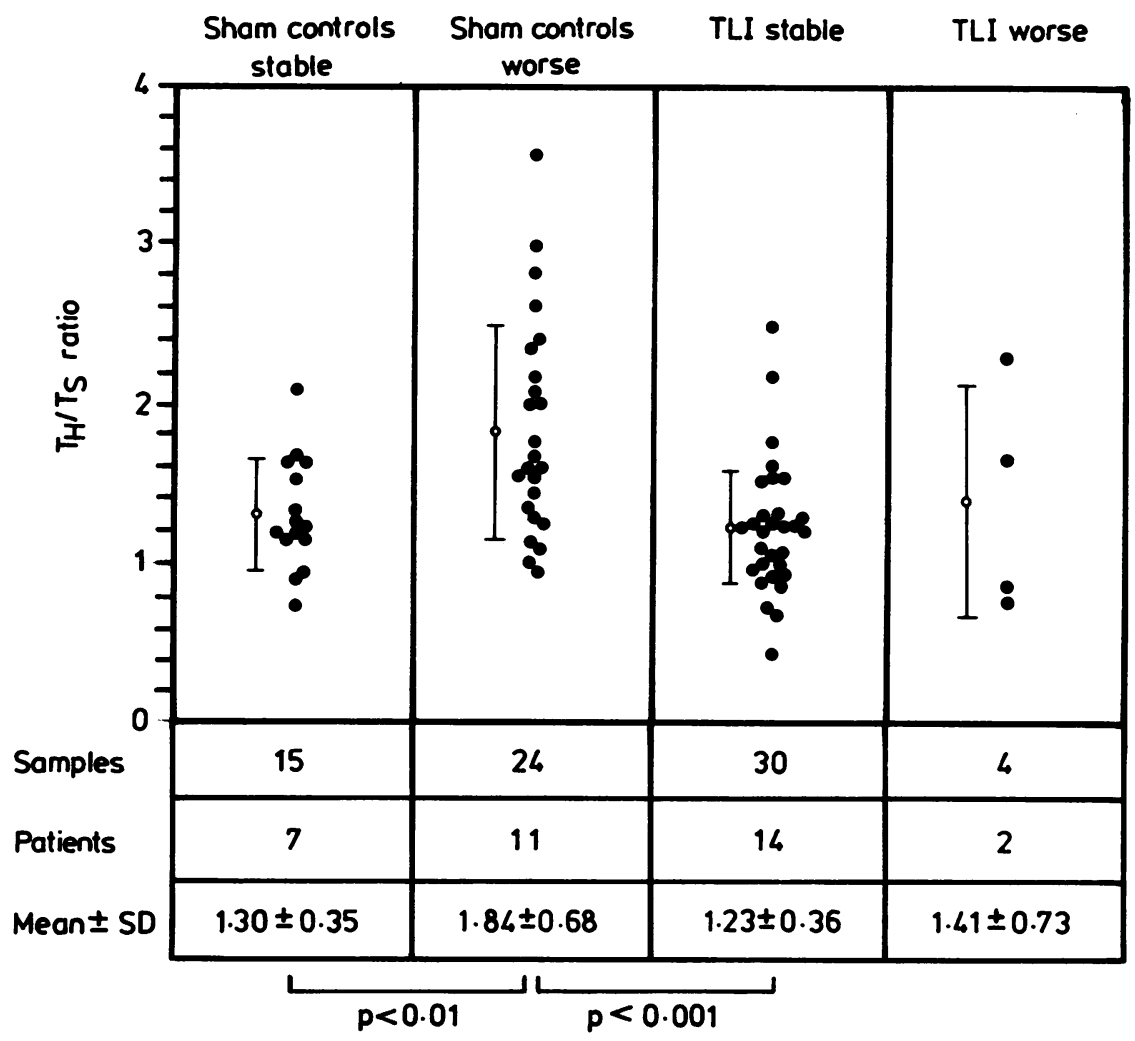

Fig Data points represent $T h / T$ s ratios of all samples from patients of each subgroup during the first year after treatment. The Th/Ts ratios of sham treated multiple sclerosis control patients who worsened clinically are significantly higher than clinically stable TLI multiple sclerosis patients $(p<0.001)$ and clinically stable sham treated multiple sclerosis controls $(p<0.01)$. 
who were clinically stable (fig). There was no significant difference in $\mathrm{Th} / \mathrm{Ts}$ ratios between the group of TLI treated patients and the group of sham controls who were clinically stable. When the serial $\mathrm{Th} / \mathrm{Ts}$ ratios from each individual patient were averaged, Th/Ts ratios of sham control multiple sclerosis patients who worsened clinically remained significantly higher $(1 \cdot 72$, SD 39$)$ than the ratios of TLI treated patients $(1.22$ SD $27, p<0.001)$ or sham control multiple sclerosis patients who were clinically stable (1.31 SD 0.14, p < 0.02).

When pre-treatment $\mathrm{Th} / \mathrm{Ts}$ ratios are also included, eight of the 11 multiple sclerosis control patients who worsened had at least one $\mathrm{Th} / \mathrm{Ts}$ ratio that was equal to or greater than two standard deviations above the mean $\mathrm{Th} / \mathrm{Ts}$ ratio of the clinically stable multiple sclerosis control and TLI treated patients.

Towards the end of the study, it was necessary to convert the analysis of patients samples from fluorescent microscopy to a FACS $\mathbf{4 4 0}$ cell sorter. To determine whether this conversion introduced a significant bias into the study, we recalculated the $p$ values after excluding all $(n=17)$ FACS analysed samples. With the FACS samples excluded, the differences between the patient groups remained significant. Sham treated patients who worsened clinically had significantly higher $\mathrm{Th} / \mathrm{Ts}$ ratios $(1 \cdot 75, \mathrm{SD}$ $0.61)$ than TLI patients $(1.22$, SD 0.58$)(\mathrm{p}<0.01)$ and sham patients who remained clinically stable $(1 \cdot 25$, SD 37) $(\mathrm{p}<0.02)$.

Observation of changes in the percentages of $\mathrm{Th}$ and Ts cells between pre- and post-treatment levels may provide some insight into the evolution of intergroup differences in $\mathrm{Th} / \mathrm{Ts}$ ratios in the post treatment period (table). Pre-treatment $T$ cell subset determinations were performed in eight TLI treated multiple sclerosis patients and 14 sham treated multiple sclerosis controls. For the group of seven sham control patients who remained clinically stable, there was no change in the Th and Ts cell percentages between pre- and post-treatment periods. For the group of seven sham treated controls who worsened clinically, the percentage of the Th cells remained unchanged; however, there was a significant fall in the percentage of Ts cells in the first year post treatment resulting in an increase in the $\mathrm{Th} / \mathrm{Ts}$ ratio. For the group of eight patients treated with TLI, the percentage of Ts cells remained unchanged. However, there was a significant fall in the percentage of Th cells in the post treatment period resulting in a significant fall in the $\mathrm{Th} / \mathrm{Ts}$ ratio. Although the number of patients who had pre- and post-treatment samples is small, the data show trends which suggest that the significantly higher post treatment $\mathrm{Th} / \mathrm{Ts}$ ratios in the control patients who worsened was the net result of a decrease in the percentage of suppressor cells in these patients in contrast to a decrease in the percentage of helper cells in TLI treated patients who remained stable.

\section{Discussion}

TLI treatment in rheumatoid arthritis patients causes lymphocytopenia, a decreased percentage of $T$ helper cells and a reduction of the Th/Ts ratio. ${ }^{7}$ We have observed similar results in chronic progressive multiple sclerosis patients treated with TLI.

In our study, TLI treatment of chronic progressive multiple sclerosis patients was associated with a significant decrease in the percentage of $T$ helper cells and a related reduction in the $\mathrm{Th} / \mathrm{Ts}$ ratio during the first year after treatment when compared to pretreatment levels in the same patients.

When our patients were grouped according to clinical outcome, the $\mathrm{Th} / \mathrm{Ts}$ ratio was significantly higher in sham treated control multiple sclerosis patients who worsened clinically during the first year after treatment than in TLI treated and sham control multiple sclerosis patients who remained clinically stable.

A number of prior studies have demonstrated abnormalities of $T$ cell subsets in active multiple sclerosis. $^{9-15}$ The results of these studies have not always been uniform. Variabilities in the results have been ascribed to the use of different anti $T$ cell monoclonal antibodies, difference in counting techniques

Table Comparison of pre-and post-treatment $T h / T s$ ratios and percentage of $T$ helper and suppressor cells in patients who had paired pre-and post- treatment samples. The percentage of $T$ suppressor cells fell significantly in sham treated control multiple sclerosis patients who worsened clinically. In contrast, in TLI treated patients the percentage of Thelper cells fell significantly while suppressor cells remained unchanged. As a result of these changes in $T$ cell subsets in the posttreatment period, the Th/Ts ratio was significantly higher for sham treated control patients who worsened clinically compared to clinically stable TLI patients $(p<0.001)$ and clinically stable sham treated multiple sclerosis controls $(p<0.005)$

\begin{tabular}{|c|c|c|c|c|c|c|}
\hline & \multicolumn{2}{|c|}{ Controls-stable } & \multicolumn{2}{|c|}{ Controls-worse } & \multicolumn{2}{|l|}{ TLI-stable } \\
\hline & Pre & Post & Pre & Post & Pre & Post \\
\hline $\begin{array}{l}\text { \% Helper (H) } \\
\text { \% Suppressor (S) } \\
\text { H/S Ratio } \\
\text { Patients } \\
\text { Samples }\end{array}$ & $\begin{array}{l}45 \cdot 86 \pm 11 \cdot 8 \\
36 \cdot 43 \pm 6 \cdot 6 \\
1 \cdot 32 \pm 0.48 \\
7 \\
7\end{array}$ & $\begin{array}{l}41 \cdot 53 \pm 11 \cdot 3 \\
33 \cdot 40 \pm 7 \cdot 4 \\
1 \cdot 30 \pm 0.35 \\
7 \\
15\end{array}$ & $\begin{array}{l}47 \cdot 0 \pm 15 \\
37.9 \pm 12 \\
1.41 \pm 0 \cdot 7 \\
7 \\
7\end{array}$ & $\begin{array}{l}50.4 \pm 10 \\
27.6 \pm 7(\mathrm{p}<0.005) \\
1.96 \pm 0.7 \\
7 \\
14\end{array}$ & $\begin{array}{l}50 \cdot 6 \pm 16 \\
31 \cdot 3 \pm 8 \\
1 \cdot 78 \pm 0 \cdot 9 \\
8 \\
8\end{array}$ & $\begin{array}{l}38.5 \pm 11(\mathrm{p}<0.005) \\
33 \cdot 2 \pm 7 \\
1.18 \pm 0.3(\mathrm{p}=0.02) \\
8 \\
17\end{array}$ \\
\hline
\end{tabular}


and the timing of sample acquisition. Increased $\mathrm{Th} / \mathrm{Ts}$ ratios associated with decreased percentages of $T$ suppressor cells have most commonly been reported in chronic progressive multiple sclerosis. ${ }^{16}$ It is not known how these abnormalities in $\mathrm{T}$ cell subsets are related to clinical progression or if they are merely an epiphenomenon; however, in general, an increase in $\mathrm{Th} / \mathrm{Ts}$ ratio is considered to be a marker of enhanced immune activity.

Our study is the first study to show that TLI reduces both the percentage of $T$ helper cells and the $T h / T s$ ratio in multiple sclerosis patients. The findings relating $\mathrm{Th} / \mathrm{Ts}$ ratios to disease activity are consistent with previous reports associating high $\mathrm{Th} / \mathrm{Ts}$ ratios with active multiple sclerosis and add further support to the concept that multiple sclerosis is an autoimmune disease.

\section{References}

1 Kotzin BL, Strober S, Engleman EG, et al. Treatment of intractable rheumatoid arthritis with total lymphoid irradiation. N Engl J Med 1981;305:969-76.

2 Trentham DE, Belli JA, Anderson RJ, et al. Clinical and immunological effects of fractional total lymphoid irradiation in refractory rheumatoid arthritis. $N$ Engl $J$ Med 1981;305:976-82.

3 Brahn E, Helfgott SM, Belli JA, et al. Total lymphoid irradiation therapy in refractory rheumatoid arthritis fifteen to forty month follow up. Arthritis Rheum 1984;27:481-8.

4 Nusslein HG, Herbst M, Manger BJ, et al. Total lymphoid irradiation in patients with refractory rheumatoid arthritis. Arthritis Rheum 1985;28:1205-10.

5 Strober S, Field E, Hoppe RT, et al. Treatment of intractable lupus nephritis with total lymphoid irradiation. Ann Int Med 1985;102:450-8.
6 Hanly JG, Hassan J, Moriarty M, et al. Lymphoid irradiation in intractable rheumatoid arthritis: a double blind, randomised study comparing 750-rad treatment with 2000 rad treatment. Arthritis Rheum 1986;29: 16-25.

7 Tanay A, Field EH, Hoppe RT, Strober S. Long term follow-up of rheumatoid arthritis patients treated with total lymphoid irradiation. Arthritis Rheum 1987;30: 1-10.

8 Cook SD, Devereux C, Troiano R, et al. Effect of total lymphoid irradiation in chronic progressive multiple sclerosis. Lancet 1986;1405-9.

9 Reinherz E, Weiner H, Hauser S, et al. Loss of suppressor $\mathrm{T}$ cells in active multiple sclerosis. $N$ Engl $J$ Med 1980;303:125-9.

10 Bach M, Phan-Dinh-Tuy F, Tournier E, et al. Deficit of suppressor $\mathrm{T}$ cells in active multiple sclerosis. Lancet 1980;ii:1221-3.

11 Paty DW, Kastrukoff L, Morgan N, Hiob L. Suppressor T-lymphocytes in multiple sclerosis: analysis of patients with acute relapsing and chronic progressive disease. Ann Neurol 1983;14:445-9.

12 Rose IM, Ginsberg AH, Rothstein TL, et al. Selective loss of a subset of $\mathrm{T}$ helper cells in active multiple sclerosis. Proc Natl Acad Sci USA 1985;82:7389-93.

13 Thompson AJ, Brazil J, Whelan CA, et al. Peripheral blood $\mathrm{T}$ lymphocyte changes in multiple sclerosis: a marker of disease progression rather than relapse? J Neurol Neurosurg Psychiatry 1986;49:905-12.

14 Ghezzi A, Zaffaroni M, Caputo D, et al. Evaluation of evoked potentials and lymphocyte subsets as possible markers of multiple sclerosis: one year follow-up of 30 patients. J Neurol Neurosurg Psychiatry 1986;49: 913-9.

15 Morimoto C, Hafler DA, Weiner HL, et al. Selective loss of the suppressor-inducer $\mathrm{T}$ cell subset in progressive multiple sclerosis. $N$ Engl J Med 1987;316:67-72.

16 Reder AT, Arnason BGW. Immunology of Multiple Sclerosis. In: JC Koetsier, ed. Handbook of Clinical Neurology Vol 3 (47) Demyelinating Diseases Chap 12. Elsevier Science Publishers BV, 1985:337-95. 